\title{
Comparison of the efficiency, safety and survival outcomes in two stem cells mobilization regimens with cyclophosphamide plus G-CSF or G-CSF alone in multiple myeloma: a meta-analysis
}

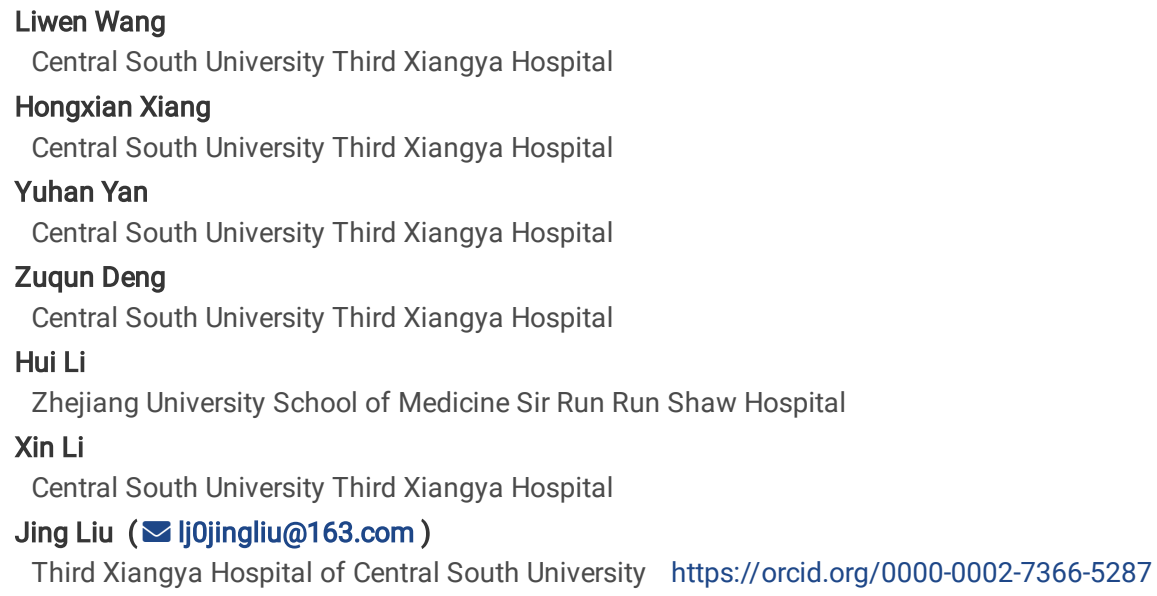




\section{Abstract}

Background Autologous stem cell transplantation as a frontline treatment for patients with multiple myeloma (MM), it requires an adequate peripheral blood stem cells (PBSC) collection before processing. Granulocyte-colony stimulating factor (G-CSF) with or without cyclophosphamide (CTX) are common regimens for PBSC mobilization, their benefits and risks are controversial.

Methods To compare the efficiency, safety, and survival outcomes between the two regimens, we conducted a meta-analysis including 18 studies with 4 prospective and 14 retrospective studies, a total of 2770 patients with MM analyzed.

Results The CTX plus G-CSF regimen had higher yields of total CD $34^{+}$cells $(\mathrm{SMD}=0.39,95 \% \mathrm{Cl}(0.30,0.49))$, and higher mobilization rates of the target $\geqslant$ $2 \times 10^{6} / \mathrm{kg}(\mathrm{OR}=3.34,95 \% \mathrm{Cl}(1.82,6.11))$ and $4 \times 10^{6} / \mathrm{kg}(\mathrm{OR}=2.16,95 \% \mathrm{Cl}(1.69,2.76))$ cells. A favorable $\mathrm{EFS}(\mathrm{HR}=0.73,95 \% \mathrm{Cl}(0.58,0.93), \mathrm{p}=0.01)$ and better 3-year EFS rate $(\mathrm{OR}=1.65,95 \% \mathrm{Cl}(1.1,2.47), \mathrm{p}=0.02)$ were also reached in the patients with CTX plus G-CSF mobilization, although the risks of admission $(\mathrm{OR}=26.49,95 \% \mathrm{Cl}(7.31,95.97))$ and fever $(\mathrm{OR}=13.66,95 \% \mathrm{Cl}(6.21,30.03))$ during mobilization were increased, the treatment-related mortality were consistent $(p=0.26)$.

Conclusions The CTX plus G-CSF regimen was superior to the G-CSF-alone regimen for PBSC mobilization in patients with MM.

\section{Background}

The estimated incidence of multiple myeloma (MM) is currently 160 000, and mortality amounts to 106000 worldwide[1]. In the United State, MM is the second common hematological malignancy, which accounts for $2.1 \%$ of all cancer-related death[2]. Survival estimates in MM are varied due to different source of the data, some randomized controlled trials (RCTs) demonstrated that the median overall survival (OS) in MM is approximately 6 years[3], and for patients with autologous stem cell transplantation (ASCT) eligible is around 8 years[4]. With further advances in the MM treatment landscape, including the development and introduction of potential new drugs, like proteasome inhibitors (PIs), immunomodulatory agents (IMiDs), antibody agents and chimeric antigen receptor T (CART) therapy, survival in MM has substantially improved in last 15 years[5]. With the sustained improvement of outcomes with new agents, there has been a topic of debate about the value of ASCT in the MM treatment modalities. However, the findings of recent large-scale RCTs still support the incorporation of ASCT into the MM treatment process[6, 7]. ASCT as frontline treatment remains the backbone in the therapy of patients with MM in the current era of novel agents[8].

Successful stem cell mobilization and adequate collection of peripheral blood stem cells (PBSC) are essential for patients with MM undergoing ASCT. Presently, the mobilization protocols used routinely in clinical practice comprise cytokines, chemo-mobilization, and the CXCR4 inhibitor Plerixafor[9]. Cyclophosphamide (CTX) combined with granulocyte-colony stimulating factor (G-CSF) or G-CSF alone are typical regimens for PBSC harvesting. The protocols with CTX plus G-CSF have been applied more than 25 years[10] while being efficient, are noted to be associated with serious treatment-related adverse effects, like neutropenic fever and hematuria[11, 12].To reduce the chemotherapeutic toxicity during mobilization, the strategy with G-CSF alone has been introduced[12]. Indeed, several types of research with small sample sizes have compared the effects of the two mobilization regimens but the conclusions still have controversies between studies[13-16]. Whether a contradiction in these data was owing to insufficient sample size or genuine heterogeneity remains unknown. Therefore, we conducted a meta-analysis to compare CTX plus G-CSF and G-CSF alone strategies in terms of the efficiency, safety of mobilization, and survival outcomes after ASCT.

\section{Methods}

\section{Search strategy}

The guidelines of the Preferred Reporting Items for Systematic Reviews and Meta-analyses[17] were followed in our study. We systematically researched the studies published in four databases, PubMed, EMBASE, the Cochrane Library, and Web of Science up to August 2020 by two independent authors. The search process was performed adopting medical subject headings (MeSH) terms, specific keywords restricted with title or abstract, and combined using the Boolean operators "AND" and "OR", search terms were appropriately adjusted for different databases. Search details can be found in supplemental file 1.

\section{Criteria for including and excluding studies}

All prospective or retrospective studies investigated the PBSC mobilization with CTX plus G-CSF and G-CSF alone in MM were eligible, a detailed description of mobilization regimens was required in all included studies. We excluded the studies as follows: 1. Granulocyte-macrophage colony-stimulating factor (GMCSF) was combined with CTX or used alone as mobilization regimens; 2 . The plerixafor was used in initial mobilization; 3 . The meta-analysis, case reports, and reviews.

\section{Literature screen}

The de-duplicated bibliography was scanned independently by two authors to exclude apparent unrelated studies. Then, the full text was reviewed, and data were extracted independently by two authors. Controversial opinions were resolved by discussion.

\section{Data collection and quality assessment}

Excel was designed to collect data including the characteristics of the studies, all parameters and values evaluating the efficiency and safety of the two specified mobilization regimens, and survival outcomes after ASCT. Also, the indirectly reported survival data from the Kaplan-Meier curve were obtained by using Engauge Digitizer software. Following data extraction, the quality of the included studies was assessed by two authors independently. The Cochrane Collaboration's risk-of-bias tool[18] was adopted for RCTs, and the Newcastle-Ottawa Scale (NOS) tool[19] was used for nonrandomized studies. 


\section{Data synthesis and analysis}

The results of the analysis were presented as standard mean differences (SMD), odds ratios (ORs), hazard ratios (HRs) and 95\% confidence intervals (Cls). For some continuous variables with medians and quartiles or extreme values, the means and standard deviations (SD) were estimated using previously published methods [20-22]. HRs from the Kaplan-Meier curves were estimated according to Tierney's approach[23] and for pooling, and the natural logarithm of median survival time ratio (MSR) was used for data processing and as an effect size for median survival data. The meta-analysis was conducted in the R. The test of Cochran's Q and Higgin's \& Thompson's ${ }^{2}$ [24] was adopted to assess heterogeneity, a fixed-effects model[25] was applied when there was no significant heterogeneity $\left(l^{2}<50 \%\right.$ or $\left.p>0.1\right)$, otherwise, a random-effects model[26] was used. Besides, subgroup analysis was conducted for exploring heterogeneity, and the sensitive analysis was also performed. Hartung-Knapp-Sidik-Jonkman (HKSJ)[27-29] method was adopted in the random-effects model for sensitive analysis. Publication bias was evaluated by Egger's test[30] when the overall effect pooled more than 10 data sets, and the funnel plot was also displayed. If publication bias was confirmed, the trim-and-fill method developed by Duval and Tweedie[31] was implemented to adjust for bias. All $p$ values were 2-sided, and $p<0.05$ was considered significant.

\section{Results}

\section{Literature retrieval and screening}

The initial search retrieved a total of 2162 studies and 813 studies were excluded due to duplication. After titles and abstracts were previewed, a further 776 irrelevant studies were excluded. Then another 18 studies were excluded after carefully reviewing the full text. Ultimately, a total of 18 studies containing 2770 MM patients met the predefined inclusion criteria. Detailed search procedures were shown in Fig. 1. The characteristics of eligible studies were summarized in Table 1. Among 18 studies, two[15, 32] studies were abstracts presented at the American Society of Hematology (ASH) and one[33] study was a sub-study that shared the same population with another RCT[34]. There are 4 prospective studies including 2 RCTs and 14 retrospective studies. Seven studies were conducted in multiple centers and 11 studies were in single centers. The dose range of CTX was $3-4 \mathrm{~g} / \mathrm{m}^{2}$ in 10 studies and $1-2 \mathrm{~g} / \mathrm{m}^{2}$ in $6 \mathrm{studies,} \mathrm{one[12]}$ study used the $6 \mathrm{~g} / \mathrm{m}^{2} \mathrm{CTX}$, and one[35] study reported 2 different CTX dose data sets. The most common does of G-CSF was $10 \mu \mathrm{g} / \mathrm{kg}$ with filgrastim or lenograstim. One study used RD as induction treatment and 8 studies adopted exclusive triplet regimens, including 2 VCD, 3 CTD, 1 BiRD, 2 RVD. The induction regimens were variant in 6 studies and 3 studies did not report the information. Additionally, 9 studies, including 969 patients who underwent ASCT after mobilization, had reported the survival outcomes between the two mobilization regimens, the features were summarized in supplementary Table 1 . 
Table 1

Summary Characteristics of Including Studies

\begin{tabular}{|c|c|c|c|c|c|c|c|c|c|c|c|c|}
\hline \multirow[t]{2}{*}{ Study } & \multirow[t]{2}{*}{$\begin{array}{l}\text { Accrual } \\
\text { period }\end{array}$} & \multirow[t]{2}{*}{ Country } & \multirow[t]{2}{*}{ Study design } & \multicolumn{2}{|c|}{ Number } & \multicolumn{2}{|c|}{$\begin{array}{l}\text { Median } \\
\text { (range) age }\end{array}$} & \multicolumn{2}{|c|}{$\begin{array}{l}\text { Gender } \\
\text { (Male/Female) }\end{array}$} & \multirow[t]{2}{*}{$\begin{array}{l}\text { Induction } \\
\text { treatment }\end{array}$} & \multicolumn{2}{|c|}{ Mobilization regimens } \\
\hline & & & & $\begin{array}{l}\text { CTX } \\
+G- \\
\text { CSF }\end{array}$ & $\begin{array}{l}\text { G- } \\
\text { CSF }\end{array}$ & $\begin{array}{l}\text { CTX+ } \\
\text { G- } \\
\text { CSF }\end{array}$ & $\stackrel{\text { G- }}{\mathrm{CSF}}$ & $\begin{array}{l}\text { CTX } \\
+\mathrm{G}- \\
\mathrm{CSF}\end{array}$ & G-CSF & & CTX+G-CSF & G-CSF \\
\hline $\begin{array}{l}\text { Bacon, W. A. } \\
\text { 2011[32] }\end{array}$ & $\begin{array}{l}2000.12- \\
2008\end{array}$ & USA & $\begin{array}{l}\text { Retrospective, } \\
\text { S }\end{array}$ & 103 & 83 & - & - & - & - & - & $\begin{array}{l}\text { CTX } 4 \mathrm{~g} / \mathrm{m}^{2} \\
+\mathrm{G}-\mathrm{CSF} \\
10 \mu \mathrm{g} / \mathrm{kg}\end{array}$ & $\begin{array}{l}\text { G-CSF } \\
10 \mu \mathrm{g} / \mathrm{kg}\end{array}$ \\
\hline $\begin{array}{l}\text { Benyamini, } \\
\text { N. 2017[36] }\end{array}$ & $\begin{array}{l}2009- \\
2013\end{array}$ & Israel & $\begin{array}{l}\text { Retrospective, } \\
\text { S }\end{array}$ & 56 & 36 & $\begin{array}{l}57.3 \\
(35- \\
68)\end{array}$ & $\begin{array}{l}59.3 \\
(41- \\
74)\end{array}$ & $39 / 17$ & $20 / 16$ & VCD & $\begin{array}{l}\text { CTX } 3 \mathrm{~g} / \mathrm{m}^{2} \\
+\mathrm{G}-\mathrm{CSF} \\
10 \mu \mathrm{g} / \mathrm{kg}\end{array}$ & $\begin{array}{l}\text { G-CSF } \\
10 \mu \mathrm{g} / \mathrm{kg}\end{array}$ \\
\hline $\begin{array}{l}\text { Chua, C. C. } \\
2018[35]\end{array}$ & $\begin{array}{l}2012.12- \\
2015.3\end{array}$ & Australia & $\begin{array}{l}\text { Retrospective, } \\
\text { M }\end{array}$ & 113 & 175 & $\begin{array}{l}60 \\
(29- \\
75)\end{array}$ & $\begin{array}{l}62 \\
(36- \\
71)\end{array}$ & $74 / 39$ & $109 / 66$ & VCD & $\begin{array}{l}\text { CTX } 1.5-2 \text { or } \\
3-4 \mathrm{~g} / \mathrm{m}^{2}+ \\
\text { filgrastim } 5 \\
\text { or } 10 \mu \mathrm{g} / \mathrm{kg} \\
\text { or } \\
\text { pegfilgrastim } \\
12 \mathrm{mg}\end{array}$ & $\begin{array}{l}\text { filgrastim } \\
10 \mathrm{or} \\
20 \mu \mathrm{g} / \mathrm{kg}\end{array}$ \\
\hline $\begin{array}{l}\text { Crusoe, E. Q. } \\
2016[16]\end{array}$ & $\begin{array}{l}2009.5- \\
2013.6\end{array}$ & Brazil & $\begin{array}{l}\text { Retrospective, } \\
\mathrm{M}\end{array}$ & 18 & 70 & $\begin{array}{l}56.5 \\
(42- \\
68)\end{array}$ & $\begin{array}{l}58 \\
(36- \\
69)\end{array}$ & $13 / 5$ & $39 / 31$ & CTD & $\begin{array}{l}\text { CTX } 1- \\
2 \mathrm{~g} / \mathrm{m}^{2}+ \\
\text { filgrastim10- } \\
15 \mu \mathrm{g} / \mathrm{kg}\end{array}$ & $\begin{array}{l}\text { filgrastim } \\
15- \\
20 \mu \mathrm{g} / \mathrm{kg}\end{array}$ \\
\hline $\begin{array}{l}\text { de la Rubia } \\
\text { J. } \\
2006[37]\end{array}$ & $\begin{array}{l}2000.1- \\
2004.12\end{array}$ & Spain & $\begin{array}{l}\text { Retrospective, } \\
\mathrm{M}\end{array}$ & 206 & 551 & $59(29-$ & 72) & $430 / 359$ & & $\begin{array}{l}\text { ViBMeCP, } \\
\text { VBAdD }\end{array}$ & $\begin{array}{l}\text { CTX } \\
1.5 \mathrm{~g} / \mathrm{m}^{2}+\mathrm{G}- \\
\operatorname{CSF} 5 \mu \mathrm{g} / \mathrm{kg}\end{array}$ & $\begin{array}{l}\text { G-CSF 10- } \\
12 \text { or } 16- \\
24 \mu \mathrm{g} / \mathrm{kg}\end{array}$ \\
\hline $\begin{array}{l}\text { Desikan, K. } \\
\text { R. 1998[12] }\end{array}$ & $\begin{array}{l}1994.6- \\
1995.7\end{array}$ & USA & $\begin{array}{l}\text { Prospective, } \\
\text { S }\end{array}$ & 22 & 22 & - & & - & & - & $\begin{array}{l}\text { CTX } 6 \mathrm{~g} / \mathrm{m}^{2} \\
+\mathrm{G}-\mathrm{CSF} \\
5 \mu \mathrm{g} / \mathrm{kg}\end{array}$ & $\begin{array}{l}\text { G-CSF } \\
16 \mu \mathrm{g} / \mathrm{kg}\end{array}$ \\
\hline $\begin{array}{l}\text { Jang, J. E. } \\
2016[38]\end{array}$ & $\begin{array}{l}2006.9- \\
2013.8\end{array}$ & Korea & $\begin{array}{l}\text { Retrospective, } \\
\mathrm{M}\end{array}$ & 117 & 62 & $\begin{array}{l}53.5 \\
(48- \\
60)\end{array}$ & $\begin{array}{l}55.5 \\
(48- \\
61)\end{array}$ & $70 / 47$ & $36 / 26$ & $\begin{array}{l}\text { Me, T, R, } \\
\text { TR, ViAD }\end{array}$ & $\begin{array}{l}\text { CTX } 3 \mathrm{~g} / \mathrm{m}^{2} \\
+\mathrm{G}-\mathrm{CSF} \\
10 \mu \mathrm{g} / \mathrm{kg}\end{array}$ & $\begin{array}{l}\text { G-CSF } \\
10 \mu \mathrm{g} / \mathrm{kg}\end{array}$ \\
\hline $\begin{array}{l}\text { Jung, S. H. } \\
2013[39]\end{array}$ & $\begin{array}{l}2004.1- \\
2011.10\end{array}$ & Korea & $\begin{array}{l}\text { Retrospective, } \\
\text { S }\end{array}$ & 48 & 6 & $55(39-$ & 69) & $29 / 25$ & & CTD & $\begin{array}{l}\text { CTX } 3 \mathrm{~g} / \mathrm{m}^{2} \\
+\mathrm{G}-\mathrm{CSF} \\
10 \mu \mathrm{g} / \mathrm{kg}\end{array}$ & $\begin{array}{l}\text { G-CSF } \\
10 \mu \mathrm{g} / \mathrm{kg}\end{array}$ \\
\hline $\begin{array}{l}\text { Kumar, S. } \\
\text { 2007[40] }\end{array}$ & $\begin{array}{l}2002.1- \\
2006.12\end{array}$ & USA & $\begin{array}{l}\text { Retrospective, } \\
\text { S }\end{array}$ & 134 & 242 & $\begin{array}{l}59 \\
(33- \\
75)\end{array}$ & $\begin{array}{l}59.3 \\
(33- \\
75)\end{array}$ & $83 / 51$ & $141 / 101$ & $\begin{array}{l}\text { D, ViAD, } \\
\text { TD, RD }\end{array}$ & $\begin{array}{l}\text { CTX } 3 \mathrm{~g} / \mathrm{m}^{2} \\
+ \text { filgrastim } \\
10 \mu \mathrm{g} / \mathrm{kg}\end{array}$ & $\begin{array}{l}\text { filgrastim } \\
10 \mu \mathrm{g} / \mathrm{kg}\end{array}$ \\
\hline $\begin{array}{l}\text { Lin, T. L. } \\
\text { 2016[41] }\end{array}$ & $\begin{array}{l}2003.1- \\
2012.12\end{array}$ & China & $\begin{array}{l}\text { Retrospective, } \\
\text { S }\end{array}$ & 78 & 10 & $\begin{array}{l}52.3 \\
d\end{array}$ & $\begin{array}{l}50.5 \\
d\end{array}$ & $37 / 39$ & $7 / 3$ & $\begin{array}{l}\text { ViAD, TD, } \\
\text { VTD, VCD }\end{array}$ & $\begin{array}{l}\text { CTX } 2 \mathrm{~g} / \mathrm{m}^{2} \\
+\mathrm{G}-\mathrm{CSF} \\
10 \mu \mathrm{g} / \mathrm{kg}\end{array}$ & $\begin{array}{l}\text { G-CSF } \\
10 \mu \mathrm{g} / \mathrm{kg}\end{array}$ \\
\hline $\begin{array}{l}\text { Mark, T. } \\
\text { 2008[42] }\end{array}$ & $\begin{array}{l}2004.12- \\
2007.4\end{array}$ & USA & $\begin{array}{l}\text { Retrospective, } \\
\text { S }\end{array}$ & 20 & 9 & $\begin{array}{l}56.3 \\
d\end{array}$ & $\begin{array}{l}62.3 \\
d\end{array}$ & $11 / 8$ & $6 / 3$ & $\mathrm{BiRD}$ & $\begin{array}{l}\text { CTX } 3 \mathrm{~g} / \mathrm{m}^{2} \\
+\mathrm{G}-\mathrm{CSF} \\
10 \mu \mathrm{g} / \mathrm{kg}\end{array}$ & $\begin{array}{l}\text { G-CSF } \\
10 \mu \mathrm{g} / \mathrm{kg}\end{array}$ \\
\hline $\begin{array}{l}\text { Nakasone, H. } \\
\text { 2009[43] }\end{array}$ & $\begin{array}{l}2000.4- \\
2007.12\end{array}$ & Japan & $\begin{array}{l}\text { Retrospective, } \\
\mathrm{M}\end{array}$ & 67 & 21 & $\begin{array}{l}54 \\
(29- \\
66)\end{array}$ & $\begin{array}{l}57 \\
(46- \\
62)\end{array}$ & $37 / 30$ & $13 / 8$ & - & $\begin{array}{l}\text { CTX } 1- \\
2 \mathrm{~g} / \mathrm{m}^{2}+ \\
400 \mu \mathrm{g} / \mathrm{m}^{2} \\
\text { filgrastim or } \\
10 \mu \mathrm{g} / \mathrm{kg} \\
\text { lenograstim }\end{array}$ & $\begin{array}{l}400 \mu \mathrm{g} / \mathrm{m}^{2} \\
\text { filgrastim } \\
\text { or } 10 \mu \mathrm{g} / \mathrm{kg} \\
\text { lenograstim }\end{array}$ \\
\hline $\begin{array}{l}\text { Silvennoinen, } \\
\text { R. 2016[34] }\end{array}$ & $\begin{array}{l}2013.1- \\
2015.2\end{array}$ & Finland & RCT, M & 34 & 35 & $\begin{array}{l}62 \\
(48- \\
69)\end{array}$ & $\begin{array}{l}63 \\
(40- \\
70)\end{array}$ & $18 / 16$ & $19 / 16$ & RVD & $\begin{array}{l}\text { CTX } 2 \mathrm{~g} / \mathrm{m}^{2} \\
+ \text { filgrastim } \\
5 \mu \mathrm{g} / \mathrm{kg}\end{array}$ & $\begin{array}{l}\text { filgrastim } \\
10 \mu \mathrm{g} / \mathrm{kg}\end{array}$ \\
\hline $\begin{array}{l}\text { Skerget, M. } \\
\text { 2016[44] }\end{array}$ & NA & Slovenia & $\begin{array}{l}\text { Prospective, } \\
\text { S }\end{array}$ & 9 & 20 & $\begin{array}{l}59 \\
(42- \\
63)\end{array}$ & $\begin{array}{l}60 \\
(35- \\
69)\end{array}$ & $5 / 4$ & $11 / 9$ & VD & $\begin{array}{l}\text { CTX } 4 \mathrm{~g} / \mathrm{m}^{2} \\
+ \text { filgrastim } \\
10 \mu \mathrm{g} / \mathrm{kg}\end{array}$ & $\begin{array}{l}\text { filgrastim } \\
10 \mu \mathrm{g} / \mathrm{kg}\end{array}$ \\
\hline $\begin{array}{l}\text { Tanimura, A. } \\
2018[13]\end{array}$ & $\begin{array}{l}1999.1- \\
2010.12\end{array}$ & Japan & $\begin{array}{l}\text { Retrospective, } \\
\text { S }\end{array}$ & 115 & 32 & $\begin{array}{l}55.1 \\
d\end{array}$ & $\begin{array}{l}58.3 \\
d\end{array}$ & $56 / 69$ & 19/13 & $\begin{array}{l}\text { ViAD, D, } \\
R, T\end{array}$ & $\begin{array}{l}\text { CTX } 4 \mathrm{~g} / \mathrm{m}^{2} \\
+400 \mu \mathrm{g} / \mathrm{m}^{2} \\
\text { filgrastim or } \\
10 \mu \mathrm{g} / \mathrm{kg} \\
\text { lenograstim }\end{array}$ & $\begin{array}{l}400 \mu \mathrm{g} / \mathrm{m}^{2} \\
\text { filgrastim } \\
\text { or } 10 \mu \mathrm{g} / \mathrm{kg} \\
\text { lenograstim }\end{array}$ \\
\hline
\end{tabular}




\begin{tabular}{|c|c|c|c|c|c|c|c|c|c|c|c|c|}
\hline \multirow[t]{2}{*}{ Study } & \multirow[t]{2}{*}{$\begin{array}{l}\text { Accrual } \\
\text { period }\end{array}$} & \multirow[t]{2}{*}{ Country } & \multirow[t]{2}{*}{ Study design } & \multicolumn{2}{|c|}{ Number } & \multicolumn{2}{|c|}{$\begin{array}{l}\text { Median } \\
\text { (range) age }\end{array}$} & \multicolumn{2}{|c|}{$\begin{array}{l}\text { Gender } \\
\text { (Male/Female) }\end{array}$} & \multirow[t]{2}{*}{$\begin{array}{l}\text { Induction } \\
\text { treatment }\end{array}$} & \multicolumn{2}{|c|}{ Mobilization regimens } \\
\hline & & & & $\begin{array}{l}\text { CTX } \\
+ \text { G- } \\
\text { CSF }\end{array}$ & $\begin{array}{l}\mathrm{G}- \\
\mathrm{CSF}\end{array}$ & $\begin{array}{l}\text { CTX+ } \\
\text { G- } \\
\text { CSF }\end{array}$ & $\begin{array}{l}\text { G- } \\
\text { CSF }\end{array}$ & $\begin{array}{l}\text { CTX } \\
+ \text { G- } \\
\text { CSF }\end{array}$ & G-CSF & & $\mathrm{CTX}+\mathrm{G}-\mathrm{CSF}$ & G-CSF \\
\hline $\begin{array}{l}\text { Tuchman, S. } \\
\text { A. } 2015[14]\end{array}$ & $\begin{array}{l}2001- \\
2008\end{array}$ & USA & $\begin{array}{l}\text { Retrospective, } \\
\text { S }\end{array}$ & 94 & 73 & $\begin{array}{l}59 \\
(34- \\
76)\end{array}$ & $\begin{array}{l}57 \\
(33- \\
73)\end{array}$ & $59 / 35$ & $29 / 44$ & $\mathrm{~T}, \mathrm{R}, \mathrm{V}$ & $\begin{array}{l}\text { СТХ 3- } \\
4 \mathrm{~g} / \mathrm{m}^{2}+ \\
\text { filgrastim } \\
10 \mu \mathrm{g} / \mathrm{kg}\end{array}$ & $\begin{array}{l}\text { filgrastim } \\
10 \mu \mathrm{gg} / \mathrm{kg}\end{array}$ \\
\hline $\begin{array}{l}\text { Valtola, J. } \\
\text { 2016[33] }\end{array}$ & $\begin{array}{l}\text { 2013.1- } \\
2014.10\end{array}$ & Finland & RCT, M & 17 & 19 & $\begin{array}{l}58 \\
(49- \\
70)\end{array}$ & $\begin{array}{l}63 \\
(52- \\
70)\end{array}$ & $10 / 7$ & $8 / 11$ & RVD & $\begin{array}{l}\text { CTX } 2 \mathrm{~g} / \mathrm{m}^{2} \\
+ \text { +filgrastim } \\
5 \mu \mathrm{g} / \mathrm{kg}\end{array}$ & $\begin{array}{l}\text { filgrastim } \\
10 \mu \mathrm{gg} / \mathrm{kg}\end{array}$ \\
\hline $\begin{array}{l}\text { Whitmill, R. } \\
\text { S. 2015[15] }\end{array}$ & $\begin{array}{l}2003- \\
2010\end{array}$ & UK & $\begin{array}{l}\text { Retrospective, } \\
\text { S }\end{array}$ & 44 & 45 & $\begin{array}{l}58 \\
(41- \\
74)\end{array}$ & $\begin{array}{l}58 \\
(38- \\
70)\end{array}$ & $55 / 34$ & & CTD & $\begin{array}{l}\text { CTX } 3 \mathrm{~g} / \mathrm{m}^{2} \\
+ \\
\text { lenogastrim } \\
10 \mu \mathrm{gg} / \mathrm{kg}\end{array}$ & $\begin{array}{l}\text { lenogastrim } \\
10 \mu \mathrm{g} / \mathrm{kg}\end{array}$ \\
\hline
\end{tabular}

CTX \& C: Cyclophosphamide; G-CSF: Granulocyte-colony stimulating factor; M: Multiple centers; S: Single centers; V: Bortezomib; T: Thalidomide; D: Dexamet Melphalan; R: Lenalidomide; Vi: Vincristine; B: Carmustine; P: Prednisone; Ad: Adriamycin; A: Doxorubicin; Bi: Clarithromycin. ${ }^{a}$ Method of quality assessment the study design which described in METHODS section. ${ }^{\mathbf{b}}$ Abstract of meeting. ${ }^{\mathbf{c}}$ Due to risk of blind. ${ }^{\mathbf{d}}$ Mean age.

\section{Mobilization efficiency}

Fourteen studies[12-16, 34-38, 40-43] including 2285 patients reported the total CD34 ${ }^{+}$cells $\left(10^{6} / \mathrm{kg}\right)$ yield between CTX plus G-CSF and G-CSF alone mobilization regimens. Due to significant heterogeneity tested $\left(\mathrm{I}^{2}=79.3 \%, \mathrm{p}<0.0001\right)$, a random-effects model was adopted and the results showed that CTX plus G-CSF regimens yield more CD34 ${ }^{+}$cells than G-CSF alone (SMD $=0.45,95 \% \mathrm{Cl}(0.24,0.66), \mathrm{p}<0.0001$, Fig. $\left.2 \mathrm{~A}\right)$. In addition, CTX subgroup analysis was also performed with random-effects model (S. Figure $1 A$ ), while the pooled effects between $3-4 \mathrm{~g} / \mathrm{m}^{2}$ and $1-2 \mathrm{~g} / \mathrm{m}^{2} \mathrm{group}$ had no difference ( $\mathrm{g}=0.84$ ). The different dose of CTX used was not the source of heterogeneity between studies. Besides, CD34 + cells amount collected on the first day was higher in CTX plus G-CSF group than G-CSF alone to a limit degree $\left(I^{2}=71.6 \%, S M D=0.66,95 \% \mathrm{Cl}(0.39,0.92), p<0.0001\right.$, Fig. 2B). Similarly, high-dose CTX treatment revealed an undifferentiated benefit compared to the low dose (SMD $=0.71$ and 0.66 , respectively, $p=0.82, S$. Figure $1 B)$.

In general, a minimal CD $34^{+}$cells target to undergo one ASCT was $\geqslant 2 \times 10^{6} / \mathrm{kg}$, successful mobilization usually defined as collection $\geqslant 4 \times 10^{6} / \mathrm{kg}$ CD $34^{+}$ cells considering two ASCTs[45]. Eleven studies[12, 13,34-39, 41-43] with 1619 patients included in the meta-analysis had compared the rate of collection $\geqslant$ $4 \times 10^{6} / \mathrm{kg} \mathrm{CD} 34^{+}$cells between the two mobilization regimens, random-effects model (Heterogeneity: $p=0.03$ ) showed that CTX plus G-CSF group had 2.8 fold higher successful mobilization rate than $\mathrm{G}-\mathrm{CSF}$ alone $(\mathrm{OR}=2.8,95 \% \mathrm{Cl}(1.82,4.29), \mathrm{p}<0.0001, \mathrm{Fig} .2 \mathrm{C})$. As regard to the rates of minimal target $\geqslant 2 \times$ $10^{6} / \mathrm{kg} \mathrm{CD} 34^{+}$cells, pooled effect also displayed an obvious advantage in CTX plus G-CSF group $\left(I^{2}=39 \%, \mathrm{OR}=3.34,95 \% \mathrm{Cl}(1.82,6.11), \mathrm{p}<0.0001, \mathrm{Fig} .2 \mathrm{D}\right)$. For subgroup analysis, different dose of CTX administration showed similar effects in both successful (S. Figure 1D) and minimal (S. Figure 1C) CD34 + cells mobilization ( $p=0.61$ and 0.34 , respectively). Additionally, apheresis times during mobilization detected smaller in the patients who received CTX plus $\mathrm{G}$-CSF regimens $\left(\mathrm{I}^{2}=90.7 \%, \mathrm{SMD}=-0.80,95 \% \mathrm{Cl}(-1.21,-0.38), \mathrm{p}=0.0002\right.$, Fig. $\left.2 \mathrm{E}\right)$. Of note, low dose of $\mathrm{CTX}$ with $1-2 \mathrm{~g} / \mathrm{m}^{2}$ displayed a more significant reduction of apheresis times than $3-4 \mathrm{~g} / \mathrm{m}^{2}$ group ( $\mathrm{SMD}=-1.47$ and -0.53 , respectively, $\mathrm{p}=0.03$, Fig. $\left.3 \mathrm{~F}\right)$.

\section{Safety of mobilization}

Certainly, 5 studies $[12,14,15,35,36]$ had coherent tendency (heterogeneity: $I^{2}=0 \%, p=0.86$ ) that CTX plus G-CSF administration demonstrated a higher risk of admission rate than G-CSF alone during mobilization $(\mathrm{OR}=26.49,95 \% \mathrm{Cl}(7.31,95.97), \mathrm{p}<0.0001, \mathrm{Fig}$. $3 \mathrm{~A})$. Similarly, fever rate was also higher in $\mathrm{CTX}$ plus G-CSF group $\left(\mathrm{OR}=13.66,95 \% \mathrm{Cl}(6.21,30.03), \mathrm{p}<0.0001\right.$, Fig. 3B), according to a fixed-effects model $\left(\mathrm{I}^{2}=0 \%, \mathrm{p}=0.74\right)$ including 9 studies[12, 14, 34-36, 38, $39,41,43]$ and 999 MM patients. Moreover, two doses of CTX showed an undifferentiated effect to fever risk in subgroup analysis ( $p=0.58, \mathbf{S}$. Figure $1 \mathrm{E})$.

\section{Response and adverse effects during ASCT}

In the present study, 9 studies (supplementary. Table 1), including 969 patients were processed to ASCT after mobilization. With regard to the response of patients after ASCT, the proportion of patients who attained very good partial response (VGPR) or better in CTX plus G-CSF (56.2\%) group were lower than GCSF alone (69.7\%) group $\left(I^{2}=0 \%, O R=0.59,95 \% \mathrm{Cl}(0.39,0.90), p=0.01, \mathrm{Fig}\right.$. 3C). However, the complete response (CR) rate and VGPR rate had no difference between the two groups $(p=0.11$ (S. Figure $2 A)$ and $p=0.98$ (S. Figure $2 B)$, respectively). About the neutrophil and platelet engraftment, the days of neutrophil recovery to $0.5 \times 10^{9} / \mathrm{L}$ and platelet recovery to $20 \times 10^{9} / \mathrm{L}$ after ASCT were similar between the two mobilization regimens $(p=0.99(\mathbf{S}$. Figure $2 \mathrm{C})$ and $0.96(\mathbf{S}$. Figure 2D), respectively). Besides, fewer units of platelet infusion needed for patients during ASCT who used CTX plus G-CSF mobilization protocols $\left(I^{2}=0 \%\right.$, SMD $=-0.77,95 \% \mathrm{Cl}(-1.11,-0.43), \mathrm{p}<0.0001$, Fig. 3D). There had no difference about the treatment-related mortality, infusion of red blood cells, days in hospital, rates of fever and pneumonitis during ASCT between the two regimens, the lymphocytes $\left(10^{9} / \mathrm{L}\right)$ recovery on day 15 after ASCT ( $p=0.26(\mathbf{S}$. Figure 2E), 0.3 (S. Figure 2F), 0.72 (S. Figure 2G), 0.07 (S. Figure 2H), 0.87 (S. Figure 2I), 0.14 (S. Figure 2J), respectively).

\section{Survival outcomes after ASCT}

There were three different survival endpoints reported in the included studies, the overall survival (OS), progression-free survival (PFS), and event-free survival (EFS) based on univariate and multivariate analysis were computed respectively. Pooled EFS without heterogeneity $\left(I^{2}=0 \%\right)$ based on 3 univariate data 
sets $[13,14,32]$ showed patients mobilized with CTX plus G-CSF had a better EFS $\left(I^{2}=0 \%, \mathrm{HR}=0.73,95 \% \mathrm{Cl}(0.56,0.93), p=0.01\right.$, Fig. $\left.4 \mathrm{~A}\right)$. However, no decisively significant tested in multivariate data $(H R=0.7, p=0.45, S$. Figure $3 A)$. Notably, patients underwent different mobilization regimens shared an equivalent OS in the meta-analysis (univariate: $\mathrm{I}^{2}=0 \%, \mathrm{HR}=0.87, \mathrm{p}=0.33$, Fig. 4B; multivariate: $\mathrm{I}^{2}=0 \%, \mathrm{HR}=0.89, \mathrm{p}=0.64, \mathrm{~S}$. Figure $3 \mathrm{~B}$ ). Similar conclusions were draw in PFS (univariate: $I^{2}=61.3 \%, H R=1.22, p=0.36$, Fig. $4 C$; multivariate: $I^{2}=51.9 \%, H R=0.57, p=0.13, S$. Figure $3 C$ ).

For the median survival time, computed MSR displayed that no significant difference was detected in the two groups about median OS, EFS, and PFS time ( $p$ $=0.97$ (S. Figure 3D), 0.45 (S. Figure 3E), and 0.91 (S. Figure 3F), respectively). The probability of OS and PFS at 1, 3 and 5 years among patients who underwent different mobilization were consistent (S. Figure 3G-L), but the patients in CTX plus G-CSF group (40.2\%) had a higher 3-year EFS rate than that in G-CSF alone (30.1\%) group $\left(I^{2}=0 \%, O R=1.65,95 \% \mathrm{Cl}(1.1,2.47), p=0.02\right.$, Fig. 4D). However, combined 1-year and 5-year EFS rates were equivalent $(p=0.12$ (S. Figure $3 \mathrm{M})$ and 0.39 (S. Figure $3 \mathrm{~N})$, respectively).

\section{Sensitive analysis and publication bias}

The results of the sensitive analysis using different models were summarized in Table 2 and supplementary Table 2, all pooled results with statistical significance were stable. Meanwhile, the forest plots recalculated the pooled effects with one study omitted each time were generated (S. Figure 4-5). The publication bias was only tested in two comparisons due to enough studies included (above 10). No publication bias detected in the comparisons of total CD $34^{+}$cells collection $(p=0.99)$. For the comparison of successful mobilization rate in the meta-analysis, a significant publication bias detected ( $\left.p=0.006\right)$, but the relationship was unaffected $(\mathrm{OR}=2.05,95 \% \mathrm{Cl}(1.31,3.21), \mathrm{p}=0.002)$ when reanalyzed by adopting the trim-and fill[31] method as described previously. The funnel plots for the two comparisons were displayed in S. Figure 6.

Table 2

Summary Results of Sensitive Analysis

\begin{tabular}{|c|c|c|c|c|c|c|c|}
\hline Parameters & $\begin{array}{l}\text { Participants in } \\
\text { Analysis }\end{array}$ & $\begin{array}{l}\text { Effect } \\
\text { Sizes * }\end{array}$ & $\begin{array}{l}\text { M-H Fixed- } \\
\text { model } \\
\text { (Effect, 95\%Cl) }\end{array}$ & $\begin{array}{l}\text { D-L Random- } \\
\text { model } \\
\text { (Effect, 95\%Cl) }\end{array}$ & $\begin{array}{l}\text { HKSJ Random- } \\
\text { model } \\
\text { (Effect, 95\%Cl) }\end{array}$ & Heterogeneity & $\begin{array}{l}\mathrm{p}- \\
\text { value }^{\mathrm{a}}\end{array}$ \\
\hline EFS-U & 482 & $\mathrm{HR}$ & $\begin{array}{l}0.73(0.56 \\
0.93)^{b}\end{array}$ & $\begin{array}{l}0.73(0.58 \\
0.93)\end{array}$ & $0.72(0.43,1.21)$ & $\begin{array}{l}\mathrm{I}^{2}: 0 \% \\
p=0.4\end{array}$ & $\begin{array}{l}p= \\
0.01\end{array}$ \\
\hline 3-year EFS & 482 & OR & $\begin{array}{l}1.65(1.10 \\
2.47)\end{array}$ & $\begin{array}{l}1.62(1.08 \\
2.44)\end{array}$ & $1.65(0.78,3.48)$ & $\begin{array}{l}\mathrm{I}^{2}: 0 \% \\
p=0.56\end{array}$ & $\begin{array}{l}p= \\
0.02\end{array}$ \\
\hline $\begin{array}{l}\text { Total CD } 34^{+} \text {cells collection } \\
\left(10^{6} / \mathrm{kg}\right)\end{array}$ & 2285 & SMD & $\begin{array}{l}0.45(0.37 \\
0.54)\end{array}$ & $\begin{array}{l}0.45(0.24 \\
0.66)\end{array}$ & $0.45(0.18,0.71)$ & $\begin{array}{l}P^{2}: 79.3 \% \\
p<0.0001\end{array}$ & $\begin{array}{l}\mathrm{p}< \\
0.0001\end{array}$ \\
\hline $\begin{array}{l}\text { CD } 34^{+} \text {cells collection on first day } \\
\left(10^{6} / \mathrm{kg}\right)\end{array}$ & 1024 & SMD & $\begin{array}{l}0.71(0.58 \\
0.64)\end{array}$ & $\begin{array}{l}0.66(0.39 \\
0.92)\end{array}$ & $0.65(0.26,1.05)$ & $\begin{array}{l}P^{2}: 71.6 \% \\
p=0.004\end{array}$ & $\begin{array}{l}\mathrm{p}< \\
0.0001\end{array}$ \\
\hline $\begin{array}{l}\text { Collection } \geqslant 4 \times 10^{6} / \mathrm{kg} \mathrm{CD} 34^{+} \\
\text {cells }\end{array}$ & 1619 & OR & $\begin{array}{l}2.25(1.77 \\
2.87)\end{array}$ & $\begin{array}{l}2.80(1.82 \\
4.29)\end{array}$ & $3.09(1.75,5.44)$ & $\begin{array}{l}P^{2}: 48.9 \% \\
p=0.03\end{array}$ & $\begin{array}{l}\mathrm{p}< \\
0.0001\end{array}$ \\
\hline $\begin{array}{l}\text { Collection } \geqslant 2 \times 10^{6} / \mathrm{kg} \mathrm{CD} 34^{+} \\
\text {cells }\end{array}$ & 556 & OR & $\begin{array}{l}3.34(1.82 \\
6.11)\end{array}$ & $\begin{array}{l}3.58(1.48 \\
8.69)\end{array}$ & $\begin{array}{l}3.38(0.82 \\
13.97)\end{array}$ & $\begin{array}{l}P^{2}: 39.0 \% \\
p=0.16\end{array}$ & $\begin{array}{l}\mathrm{p}< \\
0.0001\end{array}$ \\
\hline Days of apheresis & 1352 & SMD & $\begin{array}{l}-0.81(-0.93 \\
-0.69)\end{array}$ & $\begin{array}{l}-0.80(-1.21 \\
-0.38)\end{array}$ & $\begin{array}{l}-0.80(-1.36 \\
-0.24)\end{array}$ & $\begin{array}{l}\mathrm{P}^{2}: 90.7 \% \\
p<0.0001\end{array}$ & $\begin{array}{l}\mathrm{p}= \\
0.0002\end{array}$ \\
\hline $\begin{array}{l}\text { Rate of admission during } \\
\text { mobilization }\end{array}$ & 664 & OR & $\begin{array}{l}26.49 \\
95.97)\end{array}$ & $\begin{array}{l}26.06(7.16 \\
94.81)\end{array}$ & $\begin{array}{l}26.05(9.09 \\
74.69)\end{array}$ & $\begin{array}{l}P^{2}: 0 \% \\
p=0.86\end{array}$ & $\begin{array}{l}p< \\
0.0001\end{array}$ \\
\hline Rate of fever during mobilization & 999 & OR & $\begin{array}{l}13.66(6.21 \\
30.03)\end{array}$ & $\begin{array}{l}11.02(4.96 \\
24.48)\end{array}$ & $\begin{array}{l}10.92(5.01 \\
23.78)\end{array}$ & $\begin{array}{l}\mathrm{I}^{2}: 0 \% \\
\mathrm{p}=0.74\end{array}$ & $\begin{array}{l}\mathrm{p}< \\
0.0001\end{array}$ \\
\hline $\begin{array}{l}\text { Response to VGPR or better after } \\
\text { ASCT }\end{array}$ & 467 & OR & $\begin{array}{l}0.59(0.39 \\
0.90)\end{array}$ & $\begin{array}{l}0.60(0.39 \\
0.90)\end{array}$ & $0.60(0.44,0.81)$ & $\begin{array}{l}\mathrm{I}^{2}: 0 \% \\
\mathrm{p}=0.89\end{array}$ & $\begin{array}{l}p= \\
0.01\end{array}$ \\
\hline $\begin{array}{l}\text { Units of platelet infusions during } \\
\text { ASCT }\end{array}$ & 148 & SMD & $\begin{array}{l}-0.77(-1.11 \\
-0.43)\end{array}$ & $\begin{array}{l}-0.77(-1.11 \\
-0.43)\end{array}$ & $\begin{array}{l}-0.77(-2.49 \\
0.96)\end{array}$ & $\begin{array}{l}\mathrm{I}^{2}: 0 \% \\
\mathrm{p}=0.44\end{array}$ & $\begin{array}{l}\mathrm{p}< \\
0.0001\end{array}$ \\
\hline $\begin{array}{l}\text { M-H: Mantel-Haenszel statistical m } \\
\text { Knapp-Sidik-Jonkman adjustment } \\
\text { difference; ASCT: Autologous stem } \\
\text { CSF to G-CSF alone. a The p-value }\end{array}$ & $\begin{array}{l}\text { od for fixed ef } \\
\text { random effect } \\
\text { Il transplantat }\end{array}$ & $\begin{array}{l}\text { odel. } \\
\text {. EFS } \\
\text { R: Ve }\end{array}$ & $\begin{array}{l}\text { Simonian-Lair } \\
\text { free survival; } U \\
\text { partial respon } \\
\text { is the estimate }\end{array}$ & $\begin{array}{l}\text { atistical metho } \\
\text { ivariate; HR: H } \\
{ }^{*} \text { All effect size } \\
\text { verall effect in }\end{array}$ & $\begin{array}{l}\text { random effects } \\
\text { d ratio; OR: Odds } \\
\text { re calculated by }\end{array}$ & $\begin{array}{l}\text { del. HKSJ: Ha } \\
\text { tio; SMD: Stand } \\
\text { mparing the CT }\end{array}$ & $\begin{array}{l}\text { ng- } \\
\text { d mean } \\
\text { plus G- }\end{array}$ \\
\hline
\end{tabular}




\section{Discussion}

Generally, one big challenge for MM patients is the relapse, after each relapse, the disease will become more aggressive with shortened subsequent PFS[46]. Collections of autologous stem cells are often contaminated with myeloma cells, which might make a disputable contribution to the relapse of disease[47]. Due to facilitate more CD34 ${ }^{+}$cells yield and additional anti-myeloma effects, CTX was combined with hematopoietic growth factors (like G-CSF or GM-CSF) as a common regimen for PBSC mobilization. The dual functions of CTX might translate into a more effective mobilization and better disease control in MM patients[13,35]. There are several retrospective studies have discussed the clinical benefits and risks if CTX is administrated during mobilization, the conclusions still have arguments[48]. Additionally, only one well-designed RCTs[34] with small cases have compared the CTX plus G-CSF and G-CSF alone regimens in MM, which demonstrated that G-CSF alone was successful in most of patients to attain the defined collection target, and no difference in PFS between the study arms[33].

To the best of our knowledge, this is the first meta-analysis to compare the efficiency, safety, and survival outcomes between the two mobilization regimens for ASCT among patients with MM. As expected, patients received CTX combined G-CSF treatment had more effective mobilization, which was reflected by a higher PBSC collection in total and on the first day $(p<0.0001)$, as well as higher mobilization rates of defined PBSC collection target $(p<0.0001)$. However, the risks of admission and fever during mobilization were also increased accordingly $(p<0.0001)$. Post-transplant survival outcomes in $M M$ patients who underwent CTX plus G-CSF and G-CSF alone regimens mobilization were investigated in several studies. Tanimura et al[13] reported an improved PFS and EFS in patients who adopted the CTX plus G-CSF regimens although some trials have indicated otherwise[14, 32, 33]. The pooled results in our meta-analysis also showed a favorable EFS (HR $=0.73, p=0.01)$ and better 3-year EFS rate $(O R=1.65, p=0.02)$ in CTX plus G-CSF group, which indicated that the CTX plus $G$ CSF mobilization schedule was advantageous to benefit patients with MM remaining event-free after ASCT. However, there was no difference in OS and PFS between the MM patients who mobilized with different regimens in the meta-analysis. Notably, the dose discrepancy of CTX contributed a negligible effect for the difference according to our subgroup analysis, and the overall post-ASCT toxicity was similar in the two groups. The induction treatment with different agents was reported to have a dissimilar impact on the PBSC harvest[40,49], however, due to variant induction therapies used between studies included in our analysis, induction-therapy based subgroup analysis was not performed in the meta-analysis.

The models of mobilization of PBSC in ASCT have evolved in recent years[9]. Plerixafor, a state-of-the-art small molecule drug that is approved for PBSC mobilization as it selectively blocked the CXCR4 receptor, which participates in the trafficking and homing of stem cells to the bone marrow (BM)[50, 51]. A well-designed RCTs had confirmed the obvious advantages of plerixafor for PBSC mobilization in patients with MM[52], even as a salvage agent for typical regimens with previous mobilization failure[34]. More importantly, plerixafor also presents an anti-myeloma effect by inhibiting the MM cells homing back to BM[53]. Foreseeably, plerixafor with G-CSF will be an optimal mobilization strategy in the future. However, the high cost of plerixafor precluding its routine administration in all patients, but simply playing an on-demand role for typical mobilization protocols[54].

Although we attempted to conduct comprehensively analyzed of these included studies, some shortages and immanent limitations need to be acknowledged. There are only two RCTs with the same population included in our analysis, most of them are retrospective studies. Secondly, some pooled data were estimated from the raw values of publications based on the widely acceptable mathematical methods, it may be a partial source of heterogeneity and bias. More large-scaled RCTs are needed in the future.

\section{Conclusion}

Based on present evidence in our meta-analysis, the CTX plus G-CSF regimen had more advantages in mobilization efficacy, as well as more prolonged EFS in patients with MM after ASCT. Serious adverse effects like treatment-related mortality were consistent, although the risks of admission and fever during mobilization were increased. CTX plus G-CSF regimen was superior to G-CSF-alone regimen for PBSC mobilization in patients with MM.

\section{Declarations}

\section{Ethics approval and consent to participate}

Not applicable.

\section{Consent for publication}

Not applicable.

\section{Availability of data and materials}

All supporting data are included in the article and its additional files.

\section{Competing interests}

The authors declare that they have no competing interests.

\section{Funding}

This study is supported by National Natural Science Foundation of China (grant no. 81870166). 
Author contributions

WLW participated in all steps of the study, XHX and YYH participated in studies screen, selection and data extraction, DZQ and LH participated in the graph process, LX and LJ participated in study design and data analysis.

Acknowledgements

We thank Dr. Srinivasan Saranya (Department of Microbiology, Immunology \& Molecular Genetics, School of Medicine, UT Health San Antonio, USA) for reviewing the manuscripts.

\section{References}

1. Ferlay J, Colombet M, Soerjomataram I, Mathers C, Parkin DM, Pineros M, Znaor A, Bray F: Estimating the global cancer incidence and mortality in 2018: GLOBOCAN sources and methods. INT J CANCER 2019, 144(8):1941-1953.

2. Siegel RL, Miller KD, Jemal A: Cancer statistics, 2020. CA Cancer J Clin 2020, 70(1):7-30.

3. Durie B, Hoering A, Abidi MH, Rajkumar SV, Epstein J, Kahanic SP, Thakuri M, Reu F, Reynolds CM, Sexton R et al: Bortezomib with lenalidomide and dexamethasone versus lenalidomide and dexamethasone alone in patients with newly diagnosed myeloma without intent for immediate autologous stem-cell transplant (SWOG S0777): a randomised, open-label, phase 3 trial. LANCET 2017, 389(10068):519-527.

4. Goldschmidt H, Lokhorst HM, Mai EK, van der Holt B, Blau IW, Zweegman S, Weisel KC, Vellenga E, Pfreundschuh M, Kersten MJ et al: Bortezomib before and after high-dose therapy in myeloma: long-term results from the phase III HOVON-65/GMMG-HD4 trial. LEUKEMIA 2018, 32(2):383-390.

5. Kumar SK, Dispenzieri A, Lacy MQ, Gertz MA, Buadi FK, Pandey S, Kapoor P, Dingli D, Hayman SR, Leung N et al: Continued improvement in survival in multiple myeloma: changes in early mortality and outcomes in older patients. LEUKEMIA 2014, 28(5):1122-1128.

6. Attal M, Lauwers-Cances V, Hulin C, Leleu X, Caillot D, Escoffre M, Arnulf B, Macro M, Belhadj K, Garderet L et al: Lenalidomide, Bortezomib, and Dexamethasone with Transplantation for Myeloma. N Engl J Med 2017, 376(14):1311-1320.

7. Cavo M, Gay F, Beksac M, Pantani L, Petrucci MT, Dimopoulos MA, Dozza L, van der Holt B, Zweegman S, Oliva S et al: Autologous haematopoietic stemcell transplantation versus bortezomib-melphalan-prednisone, with or without bortezomib-lenalidomide-dexamethasone consolidation therapy, and lenalidomide maintenance for newly diagnosed multiple myeloma (EMNO2/HO95): a multicentre, randomised, open-label, phase 3 study. LANCET HAEMATOL 2020, 7(6):e456-e468.

8. Soekojo CY, Kumar SK: Stem-cell transplantation in multiple myeloma: how far have we come? Ther Adv Hemato/2019, 10:153179887.

9. Arora S, Majhail NS, Liu H: Hematopoietic Progenitor Cell Mobilization for Autologous Stem Cell Transplantation in Multiple Myeloma in Contemporary Era. Clin Lymphoma Myeloma Leuk 2019, 19(4):200-205.

10. Goldschmidt $\mathrm{H}$, Hegenbart $\mathrm{U}$, Haas R, Hunstein W: Mobilization of peripheral blood progenitor cells with high-dose cyclophosphamide (4 or $7 \mathbf{g} / \mathrm{m}^{2}$ ) and granulocyte colony-stimulating factor in patients with multiple myeloma. Bone Marrow Transplant 1996, 17(5):691-697.

11. To LB, Shepperd KM, Haylock DN, Dyson PG, Charles P, Thorp DL, Dale BM, Dart GW, Roberts MM, Sage RE et al: Single high doses of cyclophosphamide enable the collection of high numbers of hemopoietic stem cells from the peripheral blood. EXP HEMATOL 1990, 18(5):442-447.

12. Desikan KR, Barlogie B, Jagannath S, Vesole DH, Siegel D, Fassas A, Munshi N, Singhal S, Mehta J, Tindle S et al: Comparable engraftment kinetics following peripheral-blood stem-cell infusion mobilized with granulocyte colony-stimulating factor with or without cyclophosphamide in multiple myeloma. J CLIN ONCOL 1998, 16(4):1547-1553.

13. Tanimura A, Hirai R, Nakamura M, Takeshita M, Hagiwara S, Miwa A: Improved progression-free and event-free survival in myeloma patients undergoing PBSCH receiving a cyclophosphamide + G-CSF regimen than G-CSF alone. INT J HEMATOL 2018, 107(5):559-567.

14. Tuchman SA, Bacon WA, Huang LW, Long G, Rizzieri D, Horwitz M, Chute JP, Sullivan K, Morris EA, Yopp A et al: Cyclophosphamide-based hematopoietic stem cell mobilization before autologous stem cell transplantation in newly diagnosed multiple myeloma. J Clin Apher 2015, 30(3):176-182.

15. Whitmill RS, Lewis DJ, Sutton DJ, Khawaja J, Mayer G, Paneesha S, Nikolousis E, Kishore B: A retrospective single center comparison of cyclophosphamide plus G-CSF versus G-CSF alone for peripheral blood stem cell (PBSC) mobilisation following first line therapy in patients with multiple myeloma. BLOOD 2015, 126(23):1898.

16. Crusoe EQ, Higashi F, Martinez GA, Barros JC, Bellesso M, Rossato M, Marret AC, Chiattone CS, Hungria VT: Is it feasible to use granulocyte-colony stimulating factor alone to mobilize progenitor cells in multiple myeloma patients induced with a cyclophosphamide, thalidomide and dexamethasone regimen? Rev Bras Hematol Hemoter 2016, 38(4):302-309.

17. Moher D, Liberati A, Tetzlaff J, Altman DG: Preferred reporting items for systematic reviews and meta-analyses: the PRISMA statement. PLOS MED 2009, 6(7):e1000097.

18. Higgins JP, Thomas J, Chandler J, Cumpston M, Li T, Page MJ, Welch VA: Cochrane handbook for systematic reviews of interventions: John Wiley \& Sons; 2019.

19. GA W, B S, D O, J P, V W, M L, P T: The Newcastle-Ottawa Scale (NOS) for assessing the quality if nonrandomized studies in meta-analyses. In.; 2009.

20. Hozo SP, Djulbegovic B, Hozo I: Estimating the mean and variance from the median, range, and the size of a sample. BMC MED RES METHODOL 2005, 5:13.

21. Wan X, Wang W, Liu J, Tong T: Estimating the sample mean and standard deviation from the sample size, median, range and/or interquartile range. $B M C$ MED RES METHODOL 2014, 14:135. 
22. Luo D, Wan X, Liu J, Tong T: Optimally estimating the sample mean from the sample size, median, mid-range, and/or mid-quartile range. STAT METHODS MED RES 2018, 27(6):1785-1805.

23. Tierney JF, Stewart LA, Ghersi D, Burdett S, Sydes MR: Practical methods for incorporating summary time-to-event data into meta-analysis. TRIALS2007, 8:16.

24. Higgins JP, Thompson SG: Quantifying heterogeneity in a meta-analysis. STAT MED 2002, 21(11):1539-1558.

25. Mantel N: Chi-Square Tests with One Degree of Freedom; Extensions of the Mantel-Haenszel Procedure. J AM STAT ASSOC 1963, 58(303):690-700.

26. DerSimonian R, Laird N: Meta-analysis in clinical trials revisited. CONTEMP CLIN TRIALS 2015, 45(Pt A):139-145.

27. Hartung J, Knapp G: On tests of the overall treatment effect in meta-analysis with normally distributed responses. STAT MED 2001, 20(12):1771-1782.

28. Hartung J, Knapp G: A refined method for the meta-analysis of controlled clinical trials with binary outcome. STAT MED 2001, 20(24):3875-3889.

29. Sidik K, Jonkman JN: A simple confidence interval for meta-analysis. STAT MED 2002, 21(21):3153-3159.

30. Egger M, Davey SG, Schneider M, Minder C: Bias in meta-analysis detected by a simple, graphical test. BMJ 1997, 315(7109):629-634.

31. Duval S, Tweedie R: Trim and fill: A simple funnel-plot-based method of testing and adjusting for publication bias in meta-analysis. BIOMETRICS 2000, 56(2):455-463

32. Bacon WA, Long GD, Rizzieri DA, Horwitz ME, Chute JP, Sullivan KM, Yopp A, Johns A, Chao NJ, Gasparetto C: Impact of high dose cyclophosphamide on the outcome of autologous stem cell transplant in patients with newly diagnosed multiple myeloma. BLOOD 2011, 118(21).

33. Valtola J, Silvennoinen R, Ropponen A, Siitonen T, Saily M, Sankelo M, Terava V, Putkonen M, Kuittinen T, Pelkonen J et al: Blood graft cellular composition and posttransplant outcomes in myeloma patients mobilized with or without low-dose cyclophosphamide: a randomized comparison. TRANSFUSION 2016, 56(6):1394-1401.

34. Silvennoinen R, Anttila P, Saily M, Lundan T, Heiskanen J, Siitonen TM, Kakko S, Putkonen M, Ollikainen H, Terava V et al: A randomized phase II study of stem cell mobilization with cyclophosphamide+G-CSF or G-CSF alone after lenalidomide-based induction in multiple myeloma. Bone Marrow Transplant 2016, 51(3):372-376.

35. Chua CC, Lim HY, Chai KL, Ong J, Sim S, Wood C, Dickinson M, Campbell P, Hempton J, King H et al: Peripheral blood stem cell mobilisation with G-CSF alone versus G-CSF and cyclophosphamide after bortezomib, cyclophosphamide and dexamethasone induction in multiple myeloma. Bone Marrow Transplant 2018, 53(9):1116-1123.

36. Benyamini N, Avivi I, Dann EJ, Zuckerman T, Lavi N, Katz T: Comparison of engraftment following different stem cell mobilization modalities in patients with multiple myeloma treated with a uniform induction regimen containing bortezomib, cyclophosphamide and dexamethasone. ANN HEMATOL 2017, 96(3):461-467.

37. de la Rubia J, Blade J, Lahuerta JJ, Ribera JM, Martinez R, Alegre A, Garcia-Larana J, Fernandez P, Sureda A, de Arriba F et al: Effect of chemotherapy with alkylating agents on the yield of CD34+ cells in patients with multiple myeloma. Results of the Spanish Myeloma Group (GEM) Study. HAEMATOLOGICA 2006, 91(5):621-627.

38. Jang JE, Cheong JW, Kim SJ, Cho H, Suh C, Lee H, Eom HS, Yhim HY, Lee WS, Min CK et al: Selection of a mobilization regimen for multiple myeloma based on the response to induction therapy: granulocyte-colony stimulating factor (G-CSF) alone versus high-dose cyclophosphamide plus G-CSF. Leuk Lymphoma 2016, 57(6):1389-1397.

39. Jung SH, Park H, Ahn JS, Yang DH, Kim MY, Kim YK, Kim HJ, Lee JJ: Efficacy of stem cell mobilization in patients with newly diagnosed multiple myeloma after a CTD (cyclophosphamide, thalidomide, and dexamethasone) regimen. INT J HEMATOL 2013, 97(1):92-97.

40. Kumar S, Dispenzieri A, Lacy MQ, Hayman SR, Buadi FK, Gastineau DA, Litzow MR, Fonseca R, Roy V, Rajkumar SV et al: Impact of lenalidomide therapy on stem cell mobilization and engraftment post-peripheral blood stem cell transplantation in patients with newly diagnosed myeloma. LEUKEMIA 2007, 21(9):2035-2042.

41. Lin TL, Wang PN, Kuo MC, Hung YH, Chang H, Tang TC: Cyclophosphamide plus granulocyte-colony stimulating factor for hematopoietic stem cell mobilization in patients with multiple myeloma. J Clin Apher 2016, 31(5):423-428.

42. Mark T, Stern J, Furst JR, Jayabalan D, Zafar F, LaRow A, Pearse RN, Harpel J, Shore T, Schuster MW et al: Stem cell mobilization with cyclophosphamide overcomes the suppressive effect of lenalidomide therapy on stem cell collection in multiple myeloma. Biol Blood Marrow Transplant 2008, 14(7):795798.

43. Nakasone H, Kanda Y, Ueda T, Matsumoto K, Shimizu N, Minami J, Sakai R, Hagihara M, Yokota A, Oshima K et al: Retrospective comparison of mobilization methods for autologous stem cell transplantation in multiple myeloma. AM J HEMATOL 2009, 84(12):809-814.

44. Skerget M, Skopec B, Zontar D, Cernelc P: Mobilization with cyclophosphamide reduces the number of lymphocyte subpopulations in the leukapheresis product and delays their reconstitution after autologous hematopoietic stem cell transplantation in patients with multiple myeloma. RADIOL ONCOL 2016, 50(4):402-408

45. Kumar S, Giralt S, Stadtmauer EA, Harousseau JL, Palumbo A, Bensinger W, Comenzo RL, Lentzsch S, Munshi N, Niesvizky R et al: Mobilization in myeloma revisited: IMWG consensus perspectives on stem cell collection following initial therapy with thalidomide-, lenalidomide-, or bortezomibcontaining regimens. BLOOD 2009, 114(9):1729-1735.

46. Palumbo A, Anderson K: Multiple myeloma. N Engl J Med 2011, 364(11):1046-1060.

47. Kumar S, Paiva B, Anderson KC, Durie B, Landgren O, Moreau P, Munshi N, Lonial S, Blade J, Mateos MV et al: International Myeloma Working Group consensus criteria for response and minimal residual disease assessment in multiple myeloma. LANCET ONCOL 2016, 17(8):e328-e346.

48. Uy GL, Costa LJ, Hari PN, Zhang MJ, Huang JX, Anderson KC, Bredeson CN, Callander NS, Cornell RF, Perez MA et al: Contribution of chemotherapy mobilization to disease control in multiple myeloma treated with autologous hematopoietic cell transplantation. Bone Marrow Transplant 2015, 
50(12):1513-1518.

49. Nazha A, Cook R, Vogl DT, Mangan PA, Gardler M, Hummel K, Cunningham K, Luger SM, Porter DL, Schuster S et al: Stem cell collection in patients with multiple myeloma: impact of induction therapy and mobilization regimen. Bone Marrow Transplant 2011, 46(1):59-63.

50. De Clercq E: Mozobil(R) (Plerixafor, AMD3100), 10 years after its approval by the US Food and Drug Administration. Antivir Chem Chemother 2019, 27:1630018426.

51. Broxmeyer HE, Orschell CM, Clapp DW, Hangoc G, Cooper S, Plett PA, Liles WC, Li X, Graham-Evans B, Campbell TB et al: Rapid mobilization of murine and human hematopoietic stem and progenitor cells with AMD3100, a CXCR4 antagonist. J EXP MED 2005, 201(8):1307-1318.

52. DiPersio JF, Stadtmauer EA, Nademanee A, Micallef IN, Stiff PJ, Kaufman JL, Maziarz RT, Hosing C, Fruehauf S, Horwitz M et al: Plerixafor and G-CSF versus placebo and G-CSF to mobilize hematopoietic stem cells for autologous stem cell transplantation in patients with multiple myeloma. $B L O O D 2009$, 113(23):5720-5726.

53. Alsayed Y, Ngo H, Runnels J, Leleu X, Singha UK, Pitsillides CM, Spencer JA, Kimlinger T, Ghobrial JM, Jia X et al: Mechanisms of regulation of CXCR4/SDF-1 (CXCL12)-dependent migration and homing in multiple myeloma. BLOOD 2007, 109(7):2708-2717.

54. Tanhehco YC, Vogl DT, Stadtmauer EA, O'Doherty U: The evolving role of plerixafor in hematopoietic progenitor cell mobilization. TRANSFUSION2013, 53(10):2314-2326.

\section{Figures}

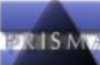

\section{PRISMA 2009 Flow Diagram}

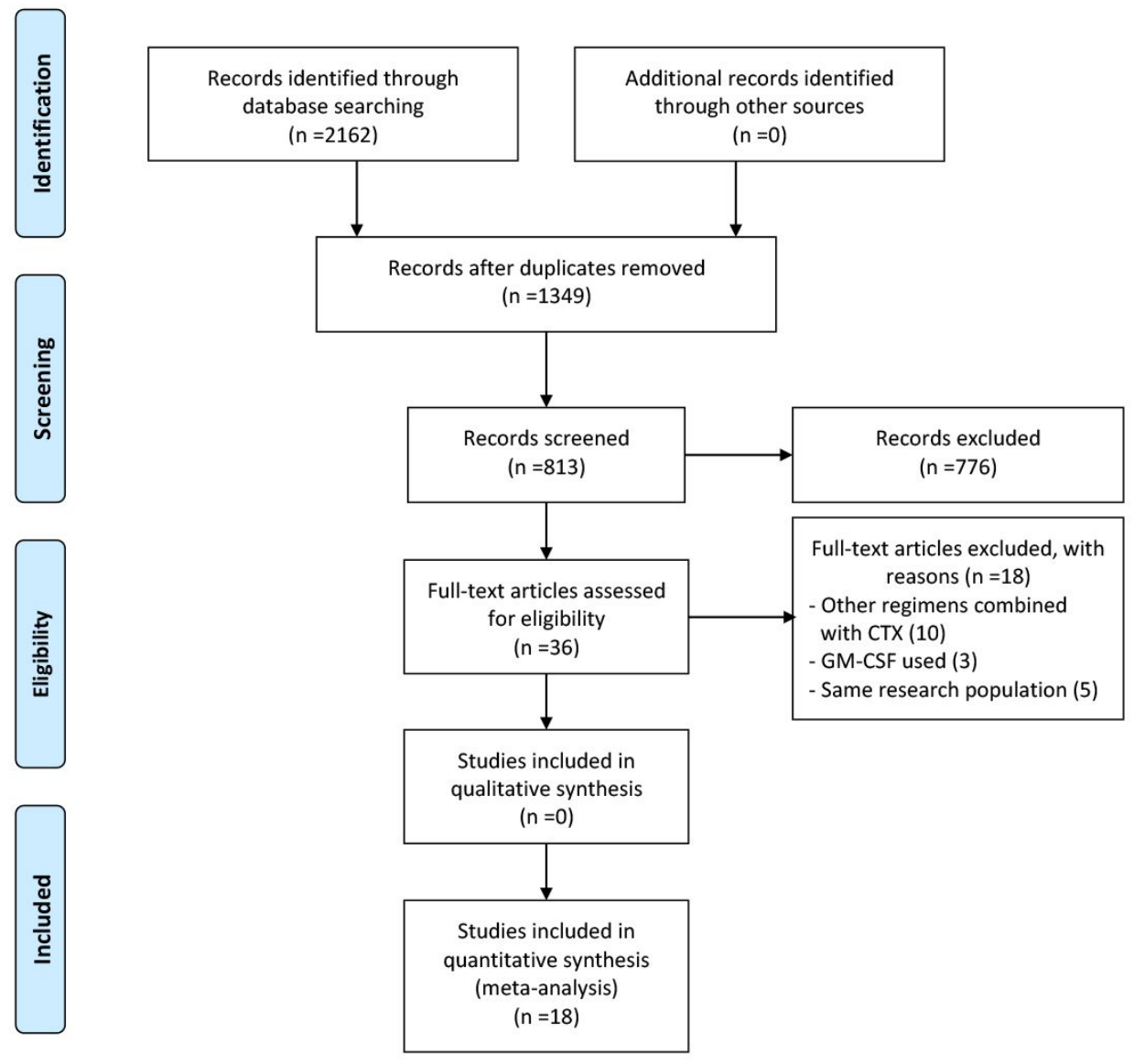

Figure 1

Flowchart of studies screen. 
A

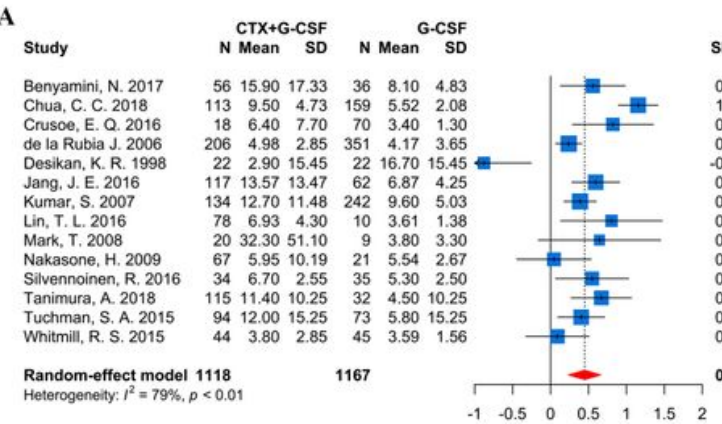

B

Study

Benyamini, N. $2017 \quad 56 \quad \begin{array}{llllll}14.60 & 9.25 & 36 & 5.20 & 2.15\end{array}$ Chua, C. C. 2018 Jang. J. E. 2016 $\begin{array}{llllll}65 & 4.07 & 3.94 & 62 & 2.90 & 2.73\end{array}$ \begin{tabular}{llllllllll} 
& 65 & 4.07 & 3.94 & 62 & 2.90 & 2.73 \\
\hline
\end{tabular} $\begin{array}{llllllll}\text { Silvennoinen, R. } 2016 & 34 & 4.00 & 2.90 & 35 & 2.70 & 2.98\end{array}$

Random-effect model 469

555

Heterogeneity. $I^{2}=72 \%, p<0.01$

CTX+G-CSF G-CSF
Study
Ee Ne Ec Nc

C

$\begin{array}{lrrrr}\text { Benyamini, N. } 2017 & 48 & 56 & 21 & 36 \\ \text { Chua, C. C. } 2018 & 107 & 113 & 129 & 159\end{array}$

Chua, C. C. 2018

de la Rubia J. 2006

Desikan, K. R. 1998

Jang, J. E. 2016

Lin, T. L. 2016

Mark, T. 2008

Nakasone, H. 2009

Silvennoinen, R. 201

Tanimura, A. 2018

Random-effect mode

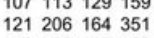

$\begin{array}{rrrr}121 & 206 & 164 & 351 \\ 18 & 22 & 17 & 22\end{array}$

$\begin{array}{llll}18 & 22 & 17 & 22 \\ 98 & 117 & 50 & 62\end{array}$

$\begin{array}{rrrr}98 & 117 & 50 & 62 \\ 39 & 48 & 2 & 6\end{array}$

$\begin{array}{rrrr}39 & 48 & 2 & 6 \\ 63 & 78 & 5 & 10\end{array}$

$\begin{array}{lllr}20 & 20 & 3 & 9\end{array}$

$\begin{array}{llll}20 & 67 & 3 & 21\end{array}$

$\begin{array}{rrrr}34 & 67 & 3 & 21 \\ 17 & 34 & 10 & 35\end{array}$

$\begin{array}{lllll}97 & 115 & 22 & 32\end{array}$

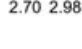

SMD $\quad 95 \% \mathrm{Cl}$ weight

$0.56[0.13 ; 0.98] \quad 7.2 \%$ $1.15[0.89 ; 1.41] \quad 8.8 \%$ $.82[0.29 ; 1.36] \quad 6.2 \%$ 0.24 [0.07: 0.41$] \quad 9.4 \%$ - 08 [-1.50; -0.26] $5.4 \%$ $0.39[0.18 ; 0.60] \quad 9.2 \%$ $0.80[0.13 ; 1.47] \quad 5.1 \%$ $0.65[-0.16 ; 1.45] \quad 4.1 \%$ $0.05[-0.45 ; 0.54] \quad 6.6 \%$ $.55[0.07 ; 1.03] \quad 6.7 \%$ $070.27,1.07] \quad 7.5 \%$ . $40[0.10 ; 0.71] \quad 8.3 \%$ $0.45[0.24 ; 0.66] 100.0 \%$

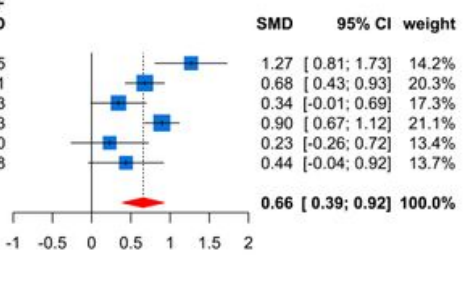
$1.27[0.81 ; 1.73] 14.2 \%$ $0.34 \quad 0.43 ; 0.99]$ [0.67:1.12] $21.1 \%$ 2. $0.0010 .39 ; 0.92100 .0 \%$

OR $\quad 95 \% \mathrm{Cl}$ weight

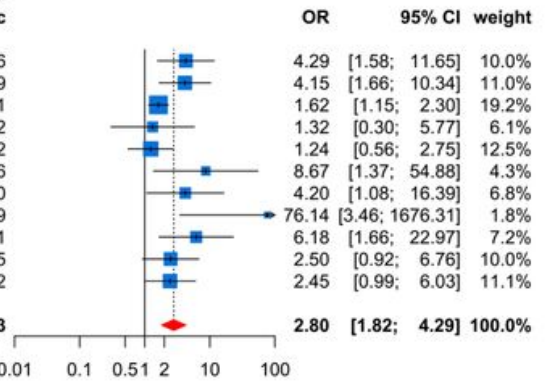

D Study $\mathrm{Ee} \mathrm{Ne} \mathrm{Ec} \mathrm{Nc}$

OR $\quad 95 \% \mathrm{Cl}$ weight

Jang, J. E. $2016 \quad 1111176162 \quad 0 \quad \ldots \quad 0.30 \quad[0.04 ; 2.58] 39.0 \%$ $\begin{array}{lllllllll}\text { Jung, S. H. } 2013 & 44 & 48 & 4 & 6 & 5.50[0.76 ; 39.92] & 5.7 \%\end{array}$ $\begin{array}{lllllllll}\text { Lin, T. L. } 2016 & 74 & 78 & 8 & 10 & 4.62[0.73 ; 29.34] & 6.9 \%\end{array}$ Nakasone, H. $2009 \quad 40 \quad 67 \quad 4 \quad 21 \quad-\quad 6.30[1.91 ; 20.77] \quad 23.4 \%$ Tanimura, A. $201810711524 \quad 32 \quad-\quad 4.46[1.52 ; 13.07] \quad 24.9 \%$

Fixed-effect model $\quad 425$ Heterogeneity: $I^{2}=39 \%, p=0.16$

131 $4.46[1.52 ; 13.07] 24.9 \%$

E Study $\quad$ CTX+G-CSF $\quad$ G-CSF \begin{tabular}{lllllll|lll} 
Benyamini, N. 2017 & 56 & 1.00 & 0.50 & 36 & 2.00 & $0.50 \quad-1.98$ & {$[-2.49 ;-1.47]$} & $10.8 \%$
\end{tabular} $\begin{array}{lllllll}\text { Chua, C. C. } 2018 & 113 & 1.00 & 0.33159 & 1.53 & 0.66 \quad-0.98[-1.23 ;-0.72] & 12.0 \%\end{array}$ Crusoe, E. Q. $2016 \quad 17 \quad 1.200 .5070 \quad 1.500 .60 \quad-0.51[-1.05 ; 0.03] 10.6 \%$

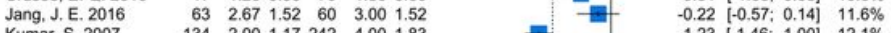
$\begin{array}{llllll}\text { Kumar, S. } 2007 \quad 134 & 2.00 & 1.17242 & 4.00 & 1.83\end{array}$ Lin, T. L. $2016 \quad \begin{array}{llllll}78 & 1.00 & 0.33 & 10 & 1.50 & 0.65 \\ & 07 & -1.32 & {[-2.00 ;-0.63]} & 9.7 \%\end{array}$

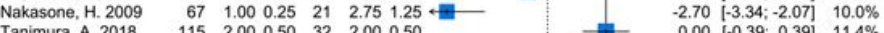
$\begin{array}{lrrrrrr}\text { Tanimura, A. } 2018 & 115 & 2.00 & 0.50 & 32 & 2.00 & 0.50\end{array}$ Tuchman, S. A. $2015 \quad 94 \quad 1.601 .91 \quad 73 \quad 2.201 .91$

Random-effect model 737

703 $-0.99[-1.45 ;-0.54] 100.0 \%$

F

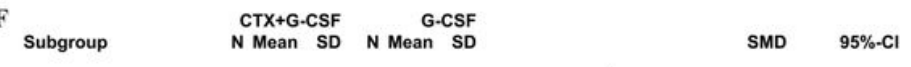

Dose $=$ CTX: $1-2 \mathrm{~g} / \mathrm{m} 2$

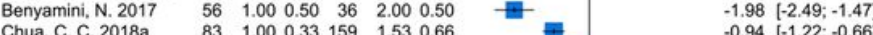

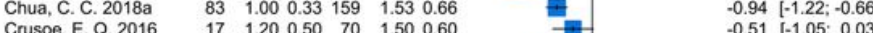
Crusoe, E. Q. 2016 - 78 - $100.33-101.500 .65$ -

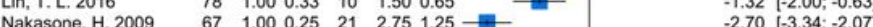

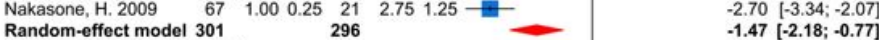
Random-effect model 301

Heterogeneity.

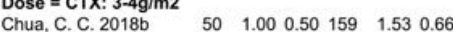
$\begin{array}{lllllll}\text { Chua, C. C. 2018b } & 50 & 1.00 & 0.50 & 159 & 1.53 & 0.66 \\ \text { Jang, J. E. } 2016 & 63 & 2.67 & 1.52 & 60 & 3.00 & 1.52\end{array}$ $\begin{array}{lrllllll}\text { Jang, J. E. } 2016 & 63 & 2.67 & 1.52 & 60 & 3.00 & 1.52 \\ \text { Kumar, S. } 2007 & 134 & 2.00 & 1.17 & 242 & 4.00 & 1.83\end{array}$ $\begin{array}{lllllll}\text { Tanimura, A. } 2018 & 115 & 2.00 & 0.50 & 32 & 2.00 & 0.50\end{array}$ $\begin{array}{lrlllll}\text { Tuchman, S. A. } 2015 & 94 & 1.60 & 1.91 & 73 & 2.20 & 1.91\end{array}$ Random-effect model $456 \quad 566$ Random-effect model 456

Test for subgroup differences: $x^{2}=4.68, \mathrm{df}=1(p=0.03)$

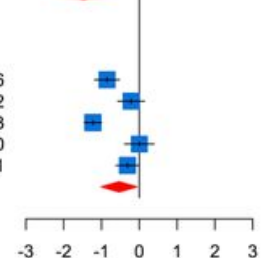

$-0.85[-1.18 ;-0.52]$ $-0.22[-0.57 ; 0.14]$ $0.00[-1.46 ;-1.00]$ $-0.31[-0.62 ;-0.01]$ $-0.53[-1.01 ;-0.05]$

Figure 2

Forest plots of mobilization efficiency between CTX plus G-CSF and G-CSF alone regimens. A: Total CD34+ cells collection. B: CD34+ cells amount collected on the first day. C: Rate of collection $\geqslant 4 x 106 / \mathrm{kg}$ CD34+ cells. D: Rate of collection $\geqslant 2 \times 106 / \mathrm{kg}$ CD34+ cells. E: Apheresis times during mobilization. F: Subgroup analysis based CTX dose for apheresis times during mobilization. 
A

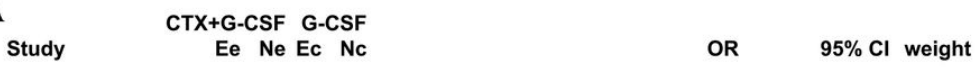

$\begin{array}{llllllll}\text { Benyamini, N. } 2017 & 9 & 56 & 0 & 36 & \longrightarrow & \longrightarrow & \\ \longrightarrow & 14.60 & {[0.82 ; 259.14]} & 25.7 \%\end{array}$

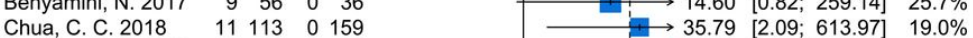

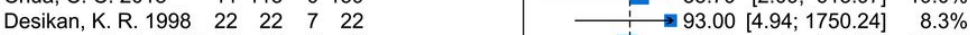
Tuchman, S. A. $201513 \quad 94 \quad 0 \quad 73 \quad \longrightarrow 24.35[1.42 ; 416.86] 24.5 \%$

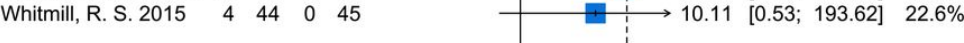

Fixed-effect model $\quad 329$ Heterogeneity: $I^{2}=0 \%, p=0.86$

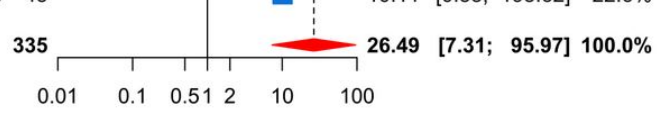

B

CTX+G-CSF G-CSF

Study $\quad \mathrm{Ee} \mathrm{Ne}$ Ec Nc

Benyamini, N. $2017 \quad 9 \quad 56 \quad 0 \quad 36$

Chua, C. C. $2018 \quad 10113 \quad 0159$

Desikan, K. R. $1998 \quad 7 \quad 7 \quad 22 \quad 1 \quad 22$

Jang, J. E. $2016 \quad 20 \quad 65 \quad 2 \quad 62$

Jung, S. H. $2013 \quad 4 \quad 4 \quad 48 \quad 0 \quad 6$

$\begin{array}{lllll}\text { Lin. T. L. } 2016 & 13 & 78 & 0 & 10\end{array}$

Nakasone, H. $2009 \quad 26 \quad 67 \quad 0 \quad 21$

Silvennoinen, R. $2016 \quad 4 \quad 34 \quad 1 \quad 35$

Tuchman, S. A. $2015 \quad 22 \quad 94 \quad 0 \quad 73$

Fixed-effect model

Heterogeneity: $I^{2}=0 \%, p=0.74$

$577 \quad 424$

OR $95 \% \mathrm{Cl}$ weight CTX+G-CSF G-CSF
Study $\quad$ Ee Ne Ec Nc

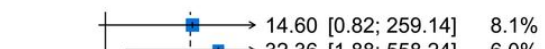

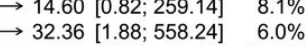
$\begin{array}{rr}\longrightarrow & 32.36[1.88 ; 558.24] \\ -\quad 9.80[1.09 ; 88.23] & 10.9 \%\end{array}$ $13.33[2.96 ; 60.00] \quad 22.6 \%$ $1.31[0.06 ; 27.36] \quad 12.7 \%$

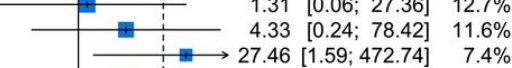
$4.53[0.48 ; 42.82] 13.9 \%$ $45.62[2.72 ; 766.25] \quad 6.8 \%$ $13.66[6.21 ; 30.03] 100.0 \%$ $\begin{array}{lllll}0.01 & 0.1 & 0.512 & 10 & 100\end{array}$

Bacon, W. A. $20115010350 \quad 83$ Jang, J. E. $2016 \quad 48 \quad 5260 \quad 62$ Tuchman, S. A. $201542 \quad 94 \quad 42 \quad 73$

Fixed-effect model

249

218 Heterogeneity: $I^{2}=0 \%, p=0.89$

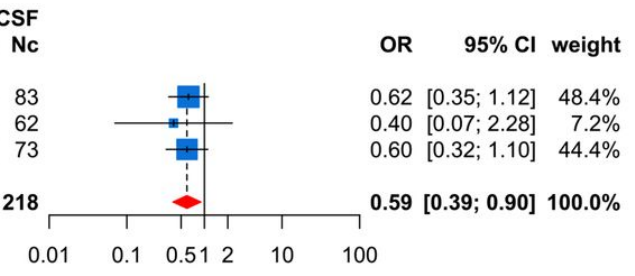

D $\begin{array}{llr}\text { CTX+G-CSF } & \text { G-CSF } \\ \text { Study } & \text { N Mean SD N Mean SD }\end{array}$

Benyamini, N. $2017 \quad 55 \quad 1.001 .25 \quad 35 \quad 3.00 \quad 3.25$ Silvennoinen, R. $201631 \quad 4.006 .0027 \quad 8.00 \quad 7.00$

Fixed-effect model 86 Heterogeneity: $I^{2}=0 \%, p=0.44$

62

SMD $\quad 95 \% \mathrm{Cl}$ weight

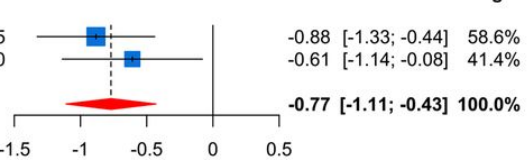

\section{Figure 3}

Forest plots of safety during mobilization and response after ASCT between CTX plus G-CSF and G-CSF alone regimens. A: Admission rate during mobilization. B: Fever rate during mobilization. C: Response to VGPR or better after ASCT. D: Units of platelet infusion needed during ASCT. 
A

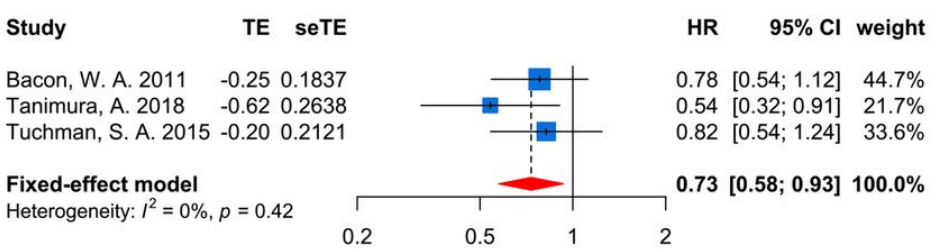

B

$\begin{array}{llll}\text { Study } & \text { TE seTE } & \text { HR } & 95 \% \mathrm{Cl} \text { weight }\end{array}$

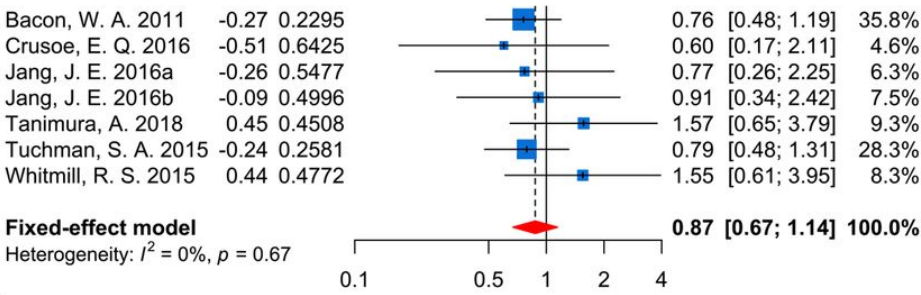

C

Study TE seTE

Crusoe, E. Q. $2016 \quad 0.020 .3961$

Jang, J. E. 2016a $\quad 0.590 .3423$

Jang, J. E. 2016b $\quad 0.710 .2472$

Nakasone, H. $2009 \quad 0.130 .3626$

Tanimura, A. $2018-0.710 .2985$

Valtola, J. 2016

Whitmill, R. S. $2015 \quad 0.390 .3200$

Random-effect model

Heterogeneity: $I^{2}=61 \%, p=0.02$

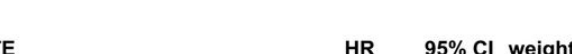

D

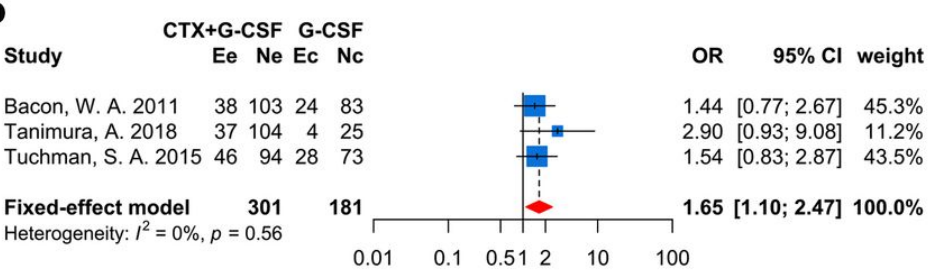

Figure 4

Survival outcomes of the patients who mobilized with CTX plus G-CSF or G-CSF alone regimens. A: Event-free survival (EFS) with univariate data. B: Overall survival (OS) with univariate data. C: Progression-free survival (PFS) with univariate data. D: 3-year EFS rate.

\section{Supplementary Files}

This is a list of supplementary files associated with this preprint. Click to download.

- Supplementaryfile1.docx

- Supplementaryfile2.docx

- Sup.Table1.CharacteristicsofPatientsUnderwentASCT.docx

- Sup.Table2.SummaryResultsofSensitiveAnalysis.docx

- S.Fig.1Subgroupanalysis.jpg

- S.Fig.2Forestforothernonsurvivaldata.jpg

- S.Fig.3Forestforothersurvivaldata.jpg

- S.Fig.4Sensitiveanalysisofnonsurvivaldata.jpg

- S.Fig.5Sensitiveanalysisofsurvivaldata.jpg

- S.Fig.6Funnelplotsforpublicationbias.jpg 